\title{
AGENTE COMUNITÁRIO DE SAÚDE: O USO DA EDUCAÇÃO EM SAÚDE COMO FACILITADORA DO CUIDADO
}

\author{
Simone Santana da Silva ${ }^{1}$ \\ Eliane dos Santos Bonfim ${ }^{2}$ \\ Monalisa Almeida da Silva ${ }^{2}$ \\ Nayanne do Santos Souza ${ }^{2}$
}

RESUMO: Este relato aborda a estratégia utilizada no planejamento e na execução do projeto de extensão "Agente comunitário de saúde: o uso da educação em saúde como facilitadora do cuidado", desenvolvido por acadêmicos de Enfermagem da Universidade do Estado da Bahia, campus VII, Senhor do Bonfim, com agentes comunitários de saúde (ACS). Após aprovação na Secretaria Municipal de Saúde do município de Senhor do Bonfim-BA, as atividades do projeto foram realizadas no período de abril a dezembro de 2013, com carga horária semanal de 12 horas. Ocorreram encontros com ACS para a discussão de temas propostos por eles, na perspectiva de facilitar suas estratégias de orientação aos usuários dos serviços de saúde. Com o projeto, foi possível compreender as especificidades existentes no desenvolvimento do trabalho desses agentes. Além disso, os discentes perceberam, na prática, como se consolida a educação em saúde e em serviços, bem como puderam participar ativamente das ações planejadas.

PALAVRAS-CHAVE: Relação comunidade-instituição. Agentes comunitários de saúde. Educação em saúde.

\section{Community health workers: using health education as health care facilitator}

\begin{abstract}
This experience report approaches the strategy used in the planning and execution of the extension project "Agente comunitário de saúde: o uso da educação em saúde como facilitadora do cuidado" (Community health workers: using health education as health care facilitator) developed by Nursing students at the University of Bahia, Campus VII, Senhor do Bonfim, state of Bahia, Brazil. After being approved by the Municipal Secretariat of Health of Senhor do Bonfim, the project was carried out from April to December 2013 for 12 hours per week. During this period, researchers and students met community health workers so different themes could be discussed with the aim of facilitating the strategies used to guide health service users. The project made possible to understand the specific characteristics of the job development of the health workers. In addition, the students could see in practice how health education and services are consolidated, they also could actively participate in the actions of the project.
\end{abstract}

KEYWORDS: Community-university relation. Community health workers. Education in health.

\footnotetext{
${ }^{1}$ Mestre em Enfermagem pela Universidade Estadual de Feira de Santana, professora auxiliar da Universidade do Estado da Bahia (campus VII - Senhor do Bonfim), membro do Grupo de Pesquisa Sobre o Cuidar em Enfermagem (GPCEnf/UNEB) (simone_ssilva1@yahoo.com.br).

${ }^{2}$ Graduandas em Enfermagem pela Universidade do Estado da Bahia, membros do Grupo de Pesquisa Sobre o Cuidar em Enfermagem (GPCEnf/UNEB) (elbonfim@hotmail.com; moninhaa.almeida@hotmail.com; nayane.nay@hotmail.com).
} 


\section{INTRODUÇÃO}

De acordo com a Constituição Federal, as universidades brasileiras gozam de autonomia didático-científica, administrativa e de gestão financeira e patrimonial (BRASIL, 1988), e devem, no exercício de suas funções, obedecer ao princípio de indissociabilidade entre ensino, pesquisa e extensão, esta última entendida como

parte indispensável do pensar e fazer universitários. É uma via de mão dupla, com trânsito assegurado à comunidade acadêmica, que encontrará, na sociedade, a oportunidade de elaboração de práxis de um conhecimento acadêmico. No retorno à universidade, docentes e discentes trarão um aprendizado que, submetido à reflexão teórica, será acrescido àqueles conhecimentos. Esse fluxo, que estabelece a troca de saberes sistematizados, acadêmico e popular, terá como consequência: a produção de conhecimento resultante do confronto com a realidade brasileira e regional, a democratização do conhecimento acadêmico e a participação efetiva da comunidade na atuação da Universidade. (FORPROEX, 1998).

Nessa perspectiva, torna-se válido evidenciar que a extensão universitária se constitui como um processo educativo, cultural e científico que articula o ensino e a pesquisa e viabiliza a relação transformadora entre universidade e sociedade (FORPROEX, 1998). Além disso, visa produzir saberes científicos, tecnológicos, artísticos e filosóficos acessíveis à população.

A Universidade do Estado da Bahia (UNEB), no Art. $3^{\circ}$ de seu Regimento Geral, defende que as atividades indissociáveis de ensino, pesquisa e extensão têm por objetivo a formação do homem como ser integral e o desenvolvimento socioeconômico, político, étnico-cultural, artístico e literário da região e do país.

A produção crítica do conhecimento científico, econômico, tecnológico, político, étnico-cultural, artístico e literário, para promover o acesso, a difusão e a socialização do saber; formação e capacitação de profissionais; e participação e contribuição no crescimento da comunidade em que se insere e, na resolução dos seus problemas. (UNEB, 2012, p. 6-7).

No âmbito da saúde coletiva, as propostas de extensão colocam-se bastante pertinentes frente à necessidade de implementação de práticas inovadoras e com vistas à reorientação da saúde. Assim, o presente relato abordará a estratégia utilizada no planejamento e na execução de um projeto de extensão desenvolvido na Universidade do Estado da Bahia (UNEB), campus VII, Senhor do Bonfim, com agentes comunitários de saúde (ACS).

O Programa Saúde da Família (PSF), uma estratégia proposta pelo Ministério da Saúde, atualmente denominada por Estratégia de Saúde da Família (ESF) (BRASIL, 2005), visa à reorientação do modelo assistencial que antes era centrado na doença, ou seja, o modelo biomédico, para uma assistência voltada à promoção e à prevenção da saúde, com a criação de vínculos efetivos entre a equipe multiprofissional e a comunidade. Tal reorientação busca proporcionar um cuidado integral e continuado, a partir da atenção básica, centrada na família, e entendida e percebida em seu ambiente físico e social, de acordo com os preceitos do Sistema Único de Saúde (SUS).

Dentro da equipe da saúde da família, o ACS é um trabalhador de destaque, pois representa o elo entre a comunidade e os demais membros da equipe (CARDOSO; NASCIMENTO, 
2007). De acordo com a Portaria 648, de 28 de março de 2006, a Política Nacional de Atenção Básica determina as atribuições dos ACS em ações de educação e de promoção de saúde.

Para Tesser et al. (2011), os ACS sabem claramente das necessidades assistenciais que a comunidade necessita, pois têm um conhecimento fundamentado sobre a vida dos indivíduos que acompanha, além do seu convívio na comunidade. Dessa forma, possuem o conhecimento de quais estratégias carece a população. Assim, na realização de estratégias de educação em saúde, os ACS necessitam ser capacitados com excelência, embora, frequentemente, se mostrem inseguros sobre as temáticas. Nesse sentido, faz-se necessário que recebam mais atenção por parte dos gestores e dos profissionais de saúde para que respaldem tais capacitações. Acrescentam-se, ainda, outros fatos que interferem no desenvolvimento das atividades do ACS, como: a falta de percepção que eles têm a respeito da sua importância na ESF; e o papel diferenciado que exercem por meio da promoção da educação em saúde extramuros, que possibilita a ida dos serviços de saúde para além da Unidade Básica de Saúde (UBS).

\section{PROPOSTA}

O projeto de extensão "Agente comunitário de saúde: o uso da educação em saúde como facilitadora do cuidado" foi desenvolvido durante o ano de 2013, utilizando como campo a unidade de saúde da zona rural do município de Senhor do Bonfim, Bahia, que buscou, por meio da ação estratégica, resultados para o processo de reflexão crítica das práticas desenvolvidas no cotidiano dos serviços de saúde pela equipe, envolvendo o cuidado no atendimento no SUS e os instrumentos de gestão disponíveis e utilizados.

O projeto procurou vislumbrar um pensar/repensar das políticas de saúde, indicando possibilidades de transformação nos espaços micropolíticos de intervenção. Além disso, objetivou proporcionar o fortalecimento de atividades que contemplem a expansão de conhecimento do ACS no seu exercício junto à comunidade, educação em saúde, contribuindo sobremaneira no cuidado e autocuidado da população. A participação de estudantes do curso de Enfermagem nas práticas de educação em saúde junto ao ACS permitiu o exercício do pensamento crítico, bem como a aproximação da realidade do PSF. O projeto procurou identificar as práticas educativas para a promoção da saúde desenvolvidas pelos ACS do município de Senhor do Bonfim-BA, ampliar as práticas educativas desenvolvidas com os ACS nas unidades de saúde da família do município e fortalecer o vínculo estabelecido entre o ACS e os usuários do PSF no cuidado.

\section{METODOLOGIA}

Trata-se de um relato de experiência a partir de encontros e diálogos com os ACS. O projeto contou com a participação de uma professora, uma bolsista e três discentes voluntárias para o seu desenvolvimento. Por tratar de um projeto de extensão, buscou-se uma parceria entre a Secretaria Municipal de Saúde de Senhor do Bonfim e a UNEB, para viabilização da proposta. Após a aprovação pelo município, priorizaram-se unidades pertencentes à zona rural, pois elas não são frequentemente contempladas com ações de extensão desenvolvidas no município. 
Para a escolha da primeira equipe, adotou-se como critérios de inclusão: equipes da ESF da zona rural que demonstrassem interesse pelas ações do projeto e que tivessem disponibilidade de tempo para os encontros.

\section{Atividades desenvolvidas}

As atividades realizadas durante a monitoria se deu no período de abril a dezembro de 2013, com carga horária semanal de 12 horas. Ocorreram encontros com os ACS para a discussão de temas propostos por eles, com a perspectiva de facilitar suas estratégias de orientação aos usuários do serviço.

\section{Capacitação}

Antes da imersão no campo de prática, ocorreu uma reunião com os monitores para apresentar o projeto e construir as atividades. Foi elaborado, nos primeiros encontros, um plano de ação, para os ACS participantes do projeto, com a atribuição de papéis para cada participante e os encontros quinzenais para execução da proposta.

Esse momento teve como objetivo despertar, entre os extensionistas, discussões e reflexões sobre o desenvolvimento da ação e suas nuances (dificuldades operacionais, políticas e sociais), com o propósito de contribuir na formação para uma aplicabilidade de ações continuadas com a comunidade.

\section{Descrição das atividades}

Os encontros com os ACS foram iniciados a partir de uma reunião, na qual se acordaram as datas e os temas a serem discutidos, bem como a contribuição de cada participante. Todos os encontros foram realizados com o suporte de material impresso confeccionados pelos monitores e ocorreram na estrutura do PSF ou em sua área de abrangência.

Entre os temas solicitados para aprofundamentos estavam: abordagem sobre álcool e outras drogas com as famílias; cuidados com a pessoa idosa; e abordagem sobre doenças sexualmente transmissíveis.

Durante e após os encontros, foi notória a participação dos ACS, com depoimentos, experiências e esclarecimento de dúvidas. Uma rica troca de saberes e experiências.

É importante evidenciar que foi acordado com os ACS que, após os encontros e discussão dos temas, eles deveriam viabilizar um retorno para a comunidade. Assim, foi realizada uma feira de saúde nas praças, encontro com grupo de idosos e em escolas.

\section{Avaliação dos monitores}

Para o acompanhamento do aproveitamento dos acadêmicos no projeto, solicitou-se que fizessem um registro sobre a experiência na participação das atividades e sua opinião sobre a importância delas em sua formação. A seguir estão dispostos alguns trechos que elucidam tais aspectos: 
A atividade extensionista proposta possui grande vantagem a todos os envolvidos, principalmente aos discentes, já que a Universidade não consegue abarcar todo o conhecimento para a atuação na prática profissional. Nesse sentido, faz-se de grande importância, pois consolida a formação dos mesmos, como também, faz com que estes busquem mais conhecimento, ao mesmo tempo em que desenvolve habilidades, passando para a comunidade um olhar diferenciado. (Monitor A).

O projeto possibilitou realizar ações educativas de promoção da saúde alcançando o objetivo proposto do projeto, que é fortalecer o conhecimento dos ACS no exercício junto à comunidade no cuidado e autocuidado da população atendida. A monitoria nos proporcionou momentos e experiências únicas, pois a inserção na comunidade permite o desenvolvimento do senso crítico, ampliação de conhecimentos que auxiliam na execução de ações de educação em saúde, na formação de profissionais (ACS) conscientes e responsáveis pelas suas práticas. (Monitor B).

Esse foi um trabalho que me permitiu avançar um pouco mais em direção ao profissional de saúde que quero ser, através dele, foi reforçada a ideia de que a educação em saúde é essencial para um bom atendimento aos pacientes, e o agente comunitário é a principal ponte que liga enfermeiros e médicos à comunidade. (Monitor C).

\section{Avanços e limites}

Não se pode deixar de mencionar a importância do desenvolvimento de ações como a proposta para a formação do discente. Diante do exposto, o projeto contribuiu para o fortalecimento de estratégias junto à Secretaria Municipal de Saúde de Senhor do Bonfim, Unidades de Saúde da Família do município e UNEB.

A participação dos discentes em todas as etapas, desde o planejamento das ações, estratégias e metodologias utilizadas, bem como a elaboração de materiais e execução das ações, oportuniza-os a desenvolverem uma sensibilidade social, por meio da aproximação com a comunidade e da compreensão das suas necessidades. $\mathrm{O}$ grupo, atualmente, já busca inserção em outras equipes do município, na perspectiva de dar continuidade às ações.

Entretanto, não se pode deixar de mencionar que existiram também limites para a execução da proposta como, por exemplo, a dificuldade de integração dos demais membros das equipes de saúde no desenvolvimento da estratégia. Além disso, a dificuldade em obter recurso financeiro viável para transporte até os locais dos encontros e aquisição de material de apoio foram outros pontos limitadores.

\section{DISCUSSÃO E CONSIDERAÇÕES FINAIS}

O ACS é um trabalhador que corrobora imensamente nas práticas de educação em saúde, se não aquele que mais dissemina esse ato na equipe multiprofissional da qual faz parte. Isso porque ele está na Unidade Básica de Saúde, realizando, no seu dia-a-dia, tal responsabilidade. Diante disso, Galavote et al. (2011) consideram o ACS como um ator social que exerce uma representatividade única, ao ser elo entre a comunidade e os serviços de 
saúde, buscando, fundamentalmente, proporcionar melhor qualidade de vida e bem-estar à população assistida por ele por meio da realização de suas atribuições.

Nessa mesma perspectiva, Campos et al. (2011) entendem que o ACS tem um papel estratégico na identificação das demandas da comunidade. A partir do momento que ele leva as atividades da UBS extramuros, antes centrada somente em um núcleo, possibilita a participação social, consequentemente, influencia os usuários a se apropriarem dos serviços ofertados.

O projeto visou contribuir para a mudança da realidade que as UBS têm acumulado no decorrer dos anos em seus atendimentos - a tradicional queixa-conduta -, visto que a realização de estratégias em saúde, viabilizadas pelo ACS, contribui para essa mudança.

Campos et al. (2011) apontam que o trabalho de educação desenvolvido pelos ACS não é valorizado pela equipe que ele integra, desmotivando-os. Justifica-se tal desvalorização em virtude do modelo que, infelizmente, tem sido seguido erroneamente pelas UBS: o curativista/ biomédico e não o da prevenção e da promoção da saúde, preconizado pela Atenção Primária.

Outro aspecto que deflagra na realização de uma educação unidirecional é o despreparo e a falta de conhecimento que os ACS têm de suas atribuições e do amplo grau de magnitude que detêm. Eles acreditam que seu trabalho estaria apenas relacionado às estratégias de educação em saúde. Ressalta-se, ainda, que muitos têm um conceito errôneo do que seja educação em saúde e promoção da saúde. Associa-se tal despreparo a uma deficiência na realização de capacitações dos profissionais de saúde e até na implementação da política de educação permanente.

A capacitação existe apenas quando eles adentraram no serviço e trata apenas de temas pontuais. Um estudo de Shimizu e Reis (2011) aponta que há o entendimento sobre a importância das capacitações, vista como uma forma de troca de conhecimento e como forma de compreender a competência específica de cada profissional, sendo necessário maior investimento de tempo das instâncias gestoras.

Para os autores, investir em capacitações é de extrema importância, pois a maioria dos profissionais não tem oportunidade de desenvolver as habilidades complexas exigidas no seu trabalho, apontando, assim, a necessidade de educação permanente em serviço para ocorrer mudanças nas práticas. Essas ações são importantes ferramentas que permitem uma melhor atuação dos profissionais e a situação da saúde no local em que esse indivíduo atua. Mesmo os ACS não cumprindo, na íntegra, a promoção da saúde, eles têm influenciado na consolidação da Atenção Primária à Saúde, por meio da educação em saúde realizada.

Frente ao exposto, e com a aplicabilidade do projeto de extensão, confirma-se que a extensão universitária aproxima a Universidade da vida comunitária, proporcionando a troca de conhecimento. As atividades extramuros permitem aos acadêmicos conhecerem a estrutura organizacional, administrativa, gerencial e funcional dos serviços de saúde, além da participação direta no cuidado à população. Permitem, também, o entendimento da legislação, da micropolítica, da compreensão de papéis e atribuições, e, ainda, sobre as reais necessidades dos sujeitos envolvidos (CONTO, 2013).

$\mathrm{Na}$ execução do projeto, foram encontradas algumas limitações para a aplicabilidade das ações de educação permanente em saúde. Tais pontos estão presentes no cotidiano da saúde brasileira, talvez por despreparo dos gestores e trabalhadores de saúde para planejar e gerir as ações (ASSIS et al., 2010). 
Parte-se da compreensão de que as dificuldades fazem parte do processo de efetivação de qualquer estratégia, o que fortalece a importância das ações de planejamento e avaliação. Tal perspectiva de compreensão permite o aprendizado dos discentes por meio da problematização e do estabelecimento de diálogo com todos os sujeitos do processo na busca pela minimização das limitações e na efetivação das estratégias na busca de um sistema de saúde mais equânime e acessível.

\section{REFERÊNCIAS}

ASSIS, M. M. A. et al. A expressão saúde, organização da rede de serviços e cuidado integral no SUS: descompassos entre o marco normativo e a legitimidade social. In: SANTOS, J. S. dos; BLIACHERIENE, A. C. (Org.). Direito à vida e à saúde: impactos orçamentário e judicial. São Paulo: Atlas, 2010, p. 237-254.

BRASIL. Constituição da República Federativa do Brasil. Brasília, DF: Senado Federal, 1988.

Ministério da Saúde. Secretaria de Vigilância em Saúde. Programa Nacional de DST e Aids. Manual de controle das doenças sexualmente transmissíveis. Brasília: Ministério da Saúde, 2005.

CAMPOS, R. O. et al. Saúde mental na atenção primária à saúde: estudo avaliativo em uma grande cidade brasileira. Ciência \& Saúde Coletiva, Rio de Janeiro, v. 16, n. 12, p. 4643-4650, jan.-dez. 2011.

CARDOSO, A. S. dos; NASCIMENTO, M. C. do. Comunicação no programa saúde da família: o agente comunitário de saúde como elo integrador entre a equipe e a comunidade. Ciência \& Saúde Coletiva, Rio de Janeiro, 2007. Disponível em: $<$ www.abrasco.org.br/cienciaesaude coletiva/artigos/artigo_int.php?id_artigo=1958>. Acesso em: 30 nov. 2013.

CONTO, F. de. A extensão universitária na faculdade de odontologia. Em Extensão, Uberlândia, v. 12, n. 2, p. 100-108, jul.-dez. 2013.

FORPROEX - Fórum de Pró-reitores de Extensão das Universidades Públicas Brasileiras. Plano Nacional de Extensão Universitária. 1998. Disponível em:

$<$ http://www.renex.org.br/documentos/Colecao-Extensao-Universitaria/01-PlanoNacional-Extensao/Plano-nacional-de-extensao-universitaria-editado.pdf $>$. Acesso em: 20 jun. 2014.

GALAVOTE, H. S. et al. Desvendando os processos de trabalho do agente comunitário de saúde nos cenários revelados na Estratégia Saúde da Família no município de Vitória (ES, Brasil). Ciência \& Saúde Coletiva, Rio de Janeiro, v.16, n. 1, p. 231-310, jan. 2011.

SHIMIZU, H. E.; REIS, L. S. As representações sociais dos trabalhadores sobre o Programa Saúde da Família. Ciência \& Saúde Coletiva, Rio de Janeiro, v.16, n. 8, p. 3461-3468, ago. 2011. 